\title{
Serum prolactin as a biomarker for the study of intracerebral dopamine effect in adult patients with phenylketonuria: a cross-sectional monocentric study
}

\author{
Eszter Juhász', Erika Kiss², Erika Simonova ${ }^{2}$, Attila Patócs $^{3}$ and Peter Reismann ${ }^{1 *}$
}

\begin{abstract}
Background: It has been previously postulated that high phenylalanine (Phe) might disturb intracerebral dopamine production, which is the main regulator of prolactin secretion in the pituitary gland. Previously, various associations between Phe and hyperprolactinemia were revealed in studies performed in phenylketonuria (PKU) children and adolescents. The aim of the present study was to clarify whether any relation between serum phenylalanine and prolactin levels can be found in adult PKU patients.
\end{abstract}

Patients and methods: We conducted a cross-sectional, monocentric study including 158 adult patients (male $n=68$, female $n=90$ ) with PKU. All patients were diagnosed during newborn screening and were treated since birth. Serum Phe, tyrosine (Tyr), prolactin (PRL), and thyroid-stimulating hormone (TSH) levels were measured, and Phe/Tyr ratio was calculated. Males and females were analyzed separately because the serum prolactin level is gender-dependent.

Results: No significant correlations were found between serum phenylalanine, tyrosine, or the Phe/Tyr ratio and serum prolactin level either in the male or in the female group.

Conclusions: In treated adult PKU patients, the serum prolactin level may not be significantly influenced by Phe or Tyr serum levels.

Keywords: Phenylketonuria, Prolactin, PKU, Dopamine

\section{Background}

Phenylketonuria (PKU, OMIM: 261600) is the most common amino acid metabolism disorder. In PKU, the deficiency of the hepatic enzyme phenylalanine hydroxylase $(\mathrm{PAH})$ causes elevated serum phenylalanine (Phe) with normal to low tyrosine (Tyr) levels. Untreated PKU leads to mental retardation, psychiatric, and various neurological disorders beyond other complications in affected patients. To achieve the best outcomes, PKU patients need a Phe-low diet and lifelong Phe-free amino acid

\footnotetext{
*Correspondence: reismann.peter@med.semmelweis-univ.hu

1 2nd Department of Medicine, Semmelweis University, Szentkirályi Street 46, Budapest 1088, Hungary
}

Full list of author information is available at the end of the article substitution. In some cases, sapropterin therapy can ease the diet limitation [1].

High phenylalanine impairs intracerebral neurotransmitter availability in multiple ways $[2,3]$. On the one hand, Phe can competitively inhibit rate-limiting enzymes in the synthesis of various transmitters (e.g., tyrosine-3 hydroxylase and tryptophan-5-hydroxylase). On the other hand, Phe blocks the transport of transmitter precursors at the neutral amino acid transporter of the blood-brain barrier (BBB) [4-6]. A recent study with rodent PKU model confirmed these pathophysiological disturbances [7]. A study by de Groot et al. showed that increased blood Phe concentrations are associated with reduced blood-to-brain Tyr transport and decreased 
intracerebral Tyr availability for neurotransmitter synthesis [8].

Obtaining information about the intracerebral neurotransmitter state in PKU is relevant, since the results can influence the treatment strategy. However, direct examination of intracerebral transmitter concentration or availability is not feasible in daily practice. For research purposes, measuring dopamine or its metabolites from intracerebral fluid is possible, but only in selective cases and not as a routine [9]. Therefore, surrogate markers are highly needed that can make a diagnostic impact of the intracerebral neurotransmitter effect in PKU.

Prolactin (luteotropic hormone, PRL), a protein hormone, is synthesized in the anterior pituitary, and its main action is the initiation and maintenance of lactation. Pituitary prolactin secretion is decisively regulated through the tonic inhibitory effect of dopamine secreted from the hypothalamic tuberoinfundibular neurons in the arcuate and periventricular nuclei (A12, 14) [10]. Intracerebral dopamine deficiency results in hyperprolactinemia [11].

In PKU, hyperphenylalaninemia can influence the intracerebral transformation of tyrosine to L-dihydroxyphenylalanine (L-DOPA), which is subsequently converted to dopamine [12]. Previous investigations presumed that elevated Phe levels can increase prolactin secretion through reduced intracerebral dopamine availability [13-15].

Clinical studies performed among children or adolescents have investigated the association between Phe and prolactin in PKU, but the results were conflicting $[6,12$, $16]$.

Therefore, our aim was to assess whether an association between Phe and PRL exists in adult PKU patients.

\section{Patients and methods Patients}

In a monocentric, cross-sectional study, 158 adult patients with PKU were consecutively enrolled between January 2014 and March 2015 at the Semmelweis University, Budapest, Hungary. All patients were diagnosed during the neonatal screening program, and their treatment was initiated from birth. Patients were regularly examined at the 2nd Department of Medicine, Semmelweis University, Budapest, Hungary.

Patients with conditions affecting prolactin secretion (pregnancy, breast feeding, thyroid or pituitary disorders, use of contraceptive pills, or drugs known to influence prolactin secretion) were excluded. All procedures followed were in accordance with the ethical standards of the responsible committee on human experimentation (Semmelweis University) and with the Helsinki Declaration of 1975, and the study was approved by the
Hungarian ethical committee (ETT TUKEB (Medical Research Council Scientific and Research Committee): reference number: 5075-2/2014/EKU). Informed consent was obtained from all patients for being included in the study.

From the 158 PKU adults, 90 patients were female and 68 were male. The mean age was $30.4 \pm 6.1$ years. According to the Hungarian PKU Guidelines, the recommended upper target Phe concentration for adulthood is $600 \mu \mathrm{mol} / \mathrm{l}$. None of the patients were treated with BH4.

\section{Methods}

All blood samples were drawn from the antecubital vein under standardized conditions in fasting state between 08:00 and 10:00 a.m. Phenylalanine and tyrosine levels were measured by API2000 LC/MS/MS at the 1st Department of Pediatrics. The serum concentration of prolactin was determined at the Central Laboratory of Semmelweis University using a chemi-immunometric assay (CMIA, Abbott Architect, Abbott Park, USA). TSH was also checked in order to rule out the TRH-induced hyperprolactinemia using this chemi-immunometric assay (CMIA, Abbott Architect, Abbott Park, USA). In a few elevated prolactin samples, PEG precipitation was used to detect macroprolactin. The reference values for prolactin were $1.4-24 \mathrm{ng} / \mathrm{ml}$ in female, $1.6-10.7 \mathrm{ng} / \mathrm{ml}$ in males; for TSH was $0.35-4.9 \mathrm{mU} / \mathrm{l}$.

\section{Statistics}

For association studies, Spearman's rho correlation coefficients was used. For analyzing the differences between different subgroups, Mann-Whitney non-parametric tests were used. Since data were not normally distributed, results are reported as median (minimum-maximum) values. All statistical analysis were performed using SPSS version 23 (IBM Corp. in Armonk, NY, USA).

\section{Results}

The median levels were as follows: Phe: 619 (124-1259) $\mu \mathrm{mol} / \mathrm{l}$; Tyr: $54(21-169) \mu \mathrm{mol} / \mathrm{l}$. The Phe/Tyr ratio was 11 (1.6-43.7). The prolactin level was $12(3-75) \mathrm{ng} / \mathrm{ml}$ in the whole studied population. All study participants had normal TSH results.

Since the serum prolactin level is gender-dependent, our patient group was divided into male and female groups and the associations were studied separately. Patient characteristics are included in Table 1. The median age of the female group was 32 (18-49) years, while in the male group, it was 31 (19-44) years. The median concentrations of Phe, Tyr, and PRL were as follows: Female group, Phe: 574 (124-1221) $\mu \mathrm{mol} / \mathrm{l}$, Tyr: 45 (21-169) $\mu \mathrm{mol} / \mathrm{l}$; PRL: 14 (5-75) ng/ml; Male group, Phe: 642 (253-1259) $\mu \mathrm{mol} / \mathrm{l}$, Tyr: 55 (24-127) $\mu \mathrm{mol} / \mathrm{l}$; PRL: 9 
Table 1 Median age and serum concentration of Phe, Tyr, Phe/Tyr, TSH, and PRL in adult patients with PKU

\begin{tabular}{|c|c|c|}
\hline & Female & Male \\
\hline Number of patients & 90 & 68 \\
\hline Age [years] (min-max) & $32(18-49)$ & $31(19-44)$ \\
\hline Phe $[\mu \mathrm{mol} / \mathrm{l}]$ (min-max) & $574(124-1221)$ & $642(253-1259)$ \\
\hline Tyr $[\mu \mathrm{mol} / \mathrm{l}]$ (min-max) & $45(21-169)$ & $55(24-127)$ \\
\hline Phe/Tyr & $11.9(1.6-43.7)$ & $11(2.4-39.5)$ \\
\hline Prolactin $[\mathrm{ng} / \mathrm{ml}]$ (min-max) & $14(5-75)$ & $9(4-47)$ \\
\hline $\mathrm{TSH}[\mathrm{mU} / \mathrm{l}]$ (min-max) & $1.5(0.6-4.3)$ & $1.1(0.4-4.9)$ \\
\hline
\end{tabular}

TSH normal range 0.35-4.9 mU/l; Prolactin: female: $1.4-24 \mathrm{ng} / \mathrm{ml}$; male: $1.6-10.7 \mathrm{ng} / \mathrm{ml}$

(4-47) $\mathrm{ng} / \mathrm{ml}$. None of the patients had prolactin-related complaints or symptoms, such as menstrual irregularity, galactorrhea, or erectile dysfunction.

It has to be mentioned that in the female group the median Phe concentration was slightly below, whereas in the male group the median Phe level was slightly above the recommended upper target Phe concentration for adulthood $(600 \mu \mathrm{mol} / \mathrm{l})$. Comparing the gender groups, males had significantly higher Phe levels $(p=0.018)$, but the Tyr level $(p=0.120)$ and the Phe/Tyr ratio $(p=0.274)$ did not differ significantly between groups.

The highest prolactin value was $75 \mathrm{ng} / \mathrm{ml}$, but this level alone without any clinical complaint does not represent a requirement for further endocrinological investigations. In samples showing a higher PRL concentration, PEG precipitation was used to detect macroprolactin, but none of these samples were found to be positive.

In the male group, neither phenylalanine nor tyrosine serum concentration, nor the Phe/Tyr ratio, showed any correlation with serum prolactin level. No association between Phe, Tyr, the Phe/Tyr ratio, and PRL was observed in female patients. The correlation coefficients are presented in Table 2. Figure 1a, b shows the relationships between Phe and PRL in the female and in the male subgroups.

Dividing our patients groups into quartiles based on the Phe level, no significant differences in prolactin concentration were observed (Table 3). In the male group, the lowest Phe-quartile was not significantly different from the highest Phe-quartile $(p=0.366)$. In the female group, the lowest Phe-quartile was also not significantly different from the highest Phe-quartile $(p=0.532)$.

\section{Discussion}

Prolactin is unique among the pituitary gland hormones because its secretion is mainly under negative tonic inhibition by hypothalamic dopamine [10]. Since dopamine biosynthesis begins from tyrosine, and its intracerebral
Table 2 Correlation coefficients and $p$ values between serum prolactin and Phe-Tyr levels in adult patients with PKU

\begin{tabular}{llllll}
\hline & Female & & & Male & \\
\cline { 2 - 3 } \cline { 5 - 6 } & $\begin{array}{l}\text { Correlation } \\
\text { coefficient }\end{array}$ & $\begin{array}{l}\boldsymbol{p} \\
\text { value }\end{array}$ & & $\begin{array}{l}\text { Correlation } \\
\text { coefficient }\end{array}$ & $\begin{array}{l}\boldsymbol{p} \\
\text { value }\end{array}$ \\
\hline Phe & -0.082 & 0.478 & 0.177 & 0.230 \\
Tyr & 0.065 & 0.580 & -0.231 & 0.114 \\
Phe/Tyr & -0.134 & 0.251 & & 0.252 & 0.084 \\
\hline
\end{tabular}

Spearman's rho correlation analysis. The significance level was set at 0.01

availability depends on phenylalanine metabolism, it was hypothesized that prolactin secretion might be altered by the hyperphenylalaninemia observed in PKU.

To date, only three studies involving a relatively small study population evaluated the relationship between Phe and PRL in PKU. On the other hand, these studies were performed in children and adolescents. Schulpis et al. found a positive correlation between Phe and PRL serum levels [16], but Denecke et al. did not confirm these associations [6]. In the study by Schulpis et al., girls with high Phe levels complained of irregular menstruation, suggesting that other underlying diseases might be responsible for the increased PRL level [16]. Recently, van Vliet et al. examined the effect of $\mathrm{BH} 4$-treatment on prolactin secretion in a few BH4-responsive male PKU patients. Their findings revealed that prolactin concentration positively correlated with blood Phe level, and the BH4-treatment lowered the Phe and PRL levels [12].

Our data obtained in a larger number of adult PKU patients showed no correlation between Phe and prolactin serum concentration. In addition, neither phenylalanine, tyrosine nor the Phe/Tyr ratio correlated with the prolactin concentration.

To interpret our results, we should take into account more factors. First, the Phe level in the total and also in the gender-specific subgroups was around the upper limit established for adults $(600 \mu \mathrm{mol} / \mathrm{l})$. One might think that very high Phe levels $(>1000 \mu \mathrm{mol} / \mathrm{l}$, representing "loose diet" patients) can increase the prolactin level. Therefore, we divided our treated patients into different quartiles based on Phe concentration and compared the PRL concentration between these subgroups. Again, no significant differences in prolactin level was detected. It should be highlighted that the studied population had some dietary restrictions that lowered serum Phe concentration. An optimal comparison would be with a group of PKU patients absolutely free of diet control.

Second, PKU itself is not a homogeneous disease, as the mutation and the enzyme activity influence the severity of PKU. However, enzyme activity determination is not 

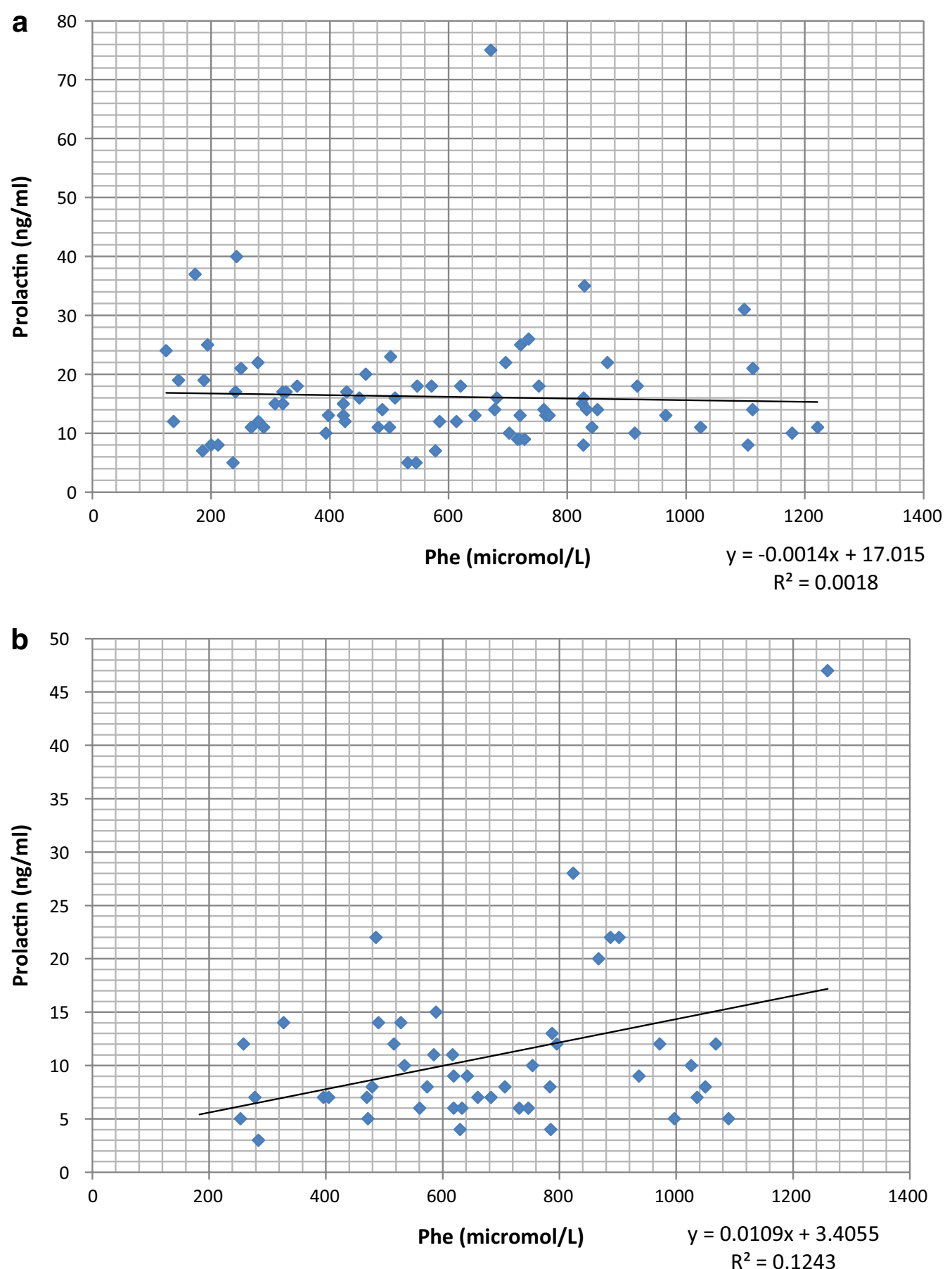

Fig. 1 a Graphical presentation of the Phe-PRL relationship in the female PKU adult subgroup. b Graphical presentation of the Phe-PRL relationship in the male PKU adult subgroup

a realistic goal in all PKU patients, whereas comparing the hormone results according to the $P A H$ gene mutation might reveal novel associations.

We also found that the tyrosine serum level did not correlate with the prolactin level. It is known that the serum
Tyr level has relatively large diurnal fluctuations [17] mediated by diet and the phenylalanine-free protein substitutes used by patients. Therefore, the calculation with one sample concentration may not be enough to detect a possible correlation with prolactin. However, using blood 
Table 3 Concentration of Phe and prolactin in patients divided into quartiles based on Phe concentration

\begin{tabular}{|c|c|c|c|c|c|c|}
\hline & \multicolumn{3}{|l|}{ Female } & \multicolumn{3}{|l|}{ Male } \\
\hline & Lowest quartile & Highest quartile & $p$ value & Lowest quartile & Highest quartile & $p$ value \\
\hline Phe $(\mu \mathrm{mol} / \mathrm{l})$ & $246(124-397)$ & $1112(1024-1121)$ & 0.000 & $284(183-404)$ & $1068(1021-1259)$ & 0.001 \\
\hline Prolactin (ng/ml) & $16(7-37)$ & $11(8-31)$ & 0.532 & $7(3-14)$ & $9(5-47)$ & 0.366 \\
\hline
\end{tabular}

Data are given as median (minimum-maximum)

samples drawn in standardized fasting conditions may eliminate the majority of these problems. Furthermore, the serum Tyr concentration cannot reflect the intracerebral Tyr concentration, which is the precursor of the intracerebral catecholamine synthesis. Hyperphenylalaninemia negatively influence dopamine utilization in the brain by competitive inhibition of the LNAA transporter and by reduced activity of DOPA decarboxylase [18]. Although there are data showing that the serum Phe level could correspondingly represent brain Phe concentration in PKU with great inter-individual variability $[19,20]$, no study performed in humans has been published that can give us an estimation of the suppressive effect of intracerebral Phe concentration on dopamine synthesis. Another limitation of our study may be that we did not take into account the menstrual cycle. However, the serum PRL level shows only minor fluctuations during the menstrual cycle, and therefore, this fluctuation may have a limited role in this current study. It should be mentioned that the strength of our study is the relatively high number of adult patients.

\section{Conclusion}

Taken together, we found no correlation between serum Phe and prolactin in a relatively large, treated adult PKU population.

\section{Abbreviations}

BBB: blood-brain barrier; BH4: tetrahydrobiopterin; LNAA: large neutral amino acid; PAH: phenylalanine hydroxylase; PEG: polyethylene glycol; Phe: phenylalanine; PKU: phenylketonuria; PRL: prolactin; TSH: thyroid-stimulating hormone; TRH: thyrotropin releasing hormone; Tyr: tyrosine.

\section{Authors' contributions}

EJ reporting the study. EK and ES conducting the study. AP proof of the study design, statistical analysis and reviewing the manuscript. PR study design, statistical analysis, and writing the manuscript. All authors read and approved the final manuscript.

\section{Author details}

${ }^{1}$ 2nd Department of Medicine, Semmelweis University, Szentkirályi Street 46, Budapest 1088, Hungary. ${ }^{2}$ 1st Department of Pediatrics, Semmelweis University, Bókay J. Street 53, Budapest 1083, Hungary. ${ }^{3}$ Hungarian Academy of Sciences and Semmelweis University "Lendület" Hereditary Endocrine Tumors Research Group, Szentkirályi Street 46, Budapest 1088, Hungary.

\section{Acknowledgements}

None.

\section{Competing interests}

The authors declare that they have no competing interests.

\section{Ethical approval}

All procedures followed were in accordance with the ethical standards of the Semmelweis University committee on human experimentation and with the Helsinki Declaration of 1975, and the study was approved by the Hungarian ethical committee (ETTTUKEB (Medical Research Council Scientific and Research Committee): reference number: 5075-2/2014/EKU). Informed consent was obtained from all patients for being included in the study.

Received: 22 December 2015 Accepted: 11 April 2016

Published online: 11 May 2016

\section{References}

1. Blau N, Erlandsen $\mathrm{H}$. The metabolic and molecular bases of tetrahydrobiopterin-responsive phenylalanine hydroxylase deficiency. Mol Genet Metab. 2004;82:101-11.

2. Güttler F, Lou H. Dietary problems of phenylketonuria: effect on CNS transmitter and their possible role in behaviour and neurophysiological function. J Inherit Metab Dis. 1986;9(Suppl. 2):169-77.

3. Velema M, Boot E, Engelen M, Hollak C. Parkinsonism in phenylketonuria: a consequence of dopamine depletion? JIMD Report. 2015;20:35-8.

4. McKean CM. The effect of high phenylalanine concentration on serotonin and catecholamine metabolism in the human brain. Brain Res. 1972:47:469-76.

5. Curtius HC, Niederwieser A, Viscontini M, Leimbacher W, Wegmann $H$, Blehova B, Rey F, Schaub J, Schmidt H. Serotonin and dopamine synthesis in phenylketonuria. Adv Exp Med Biol. 1981;133:277-91.

6. Denecke J, Schlegel W, Koch HG, Feldmann R, Harms E, Weglage J. Prolactin, a marker for cerebral dopamine deficiency in patients suffering from phenylketonuria (PKU)? J Inherit Metab Dis. 2000;23:849-51.

7. van Vliet $D$, Bruinenberg VM, Mazzola PN, van Faassen MHJR, de Blaauw P, Kema IP, Heiner-Fokkema MR, van Anholt RD, van der Zee EA, van Spronsen FJ. Large neutral amino acid supplementation exerts its effect through three synergistic mechanisms: proof of principle in phenylketonuria mice. PLoS One. 2015;10(12):e0143833.

8. de Groot MJ, Hoeksma M, Reijngoud DJ, de Valk HW, Paans AM, Suaer PJ, van Spronsen FJ. Phenylketonuria: reduced tyrosine brain influx relates to reduced cerebral protein synthesis. Orphanet J Rare Dis. 2013;8:133.

9. Burlina AB, Bonafe L, Ferrari V, Suppiej A, Zacchello F, Burlina AP. Measurement of neurotransmitter metabolites in the cerebrospinal fluid of phenylketonuric patients under dietary treatment. J Inherit Metab Dis. 2000:23:313-6.

10. Ben-Jonathan N, Hnasko R. Dopamine as a Prolactin (PRL) inhibitor. Endocr Rev. 2001;22:724-63.

11. Spada M, Ferraris S, Ferrero GB, Sartore M, Lanza C, PErfetto F, de Sanctis L, Dompe C, Blau N, Ponzone A. Monitoring treatment in tetrahydrobiopterin deficiency by serum prolactin. J Inherit Metab Dis. 1996;19:231-3.

12. van Vliet $D$, Anjema K, Jahja R, de Groot MJ, Liemburg GB, HeinerFokkema MR, van der Zee EA, Derks TGJ, Kema IP, van Spronsen FJ. BH4 treatment in $\mathrm{BH} 4$-responsive $\mathrm{PKU}$ patients: preliminary data on blood prolactin concentrations suggest increased cerebral dopamin concentrations. Mol Genet Metab. 2015;114:29-33. 
13. Carlson HE, Hyman DB, Blitzer MG. Evidence for an intracerebral action of phenylalanine in stimulation of prolactin secretion: interaction of large neutral amino acids. J Clin Endocrinol Metab. 1990;70:814-6.

14. Carlson HE, Hyman DB, Bauman C, Koch R. Prolactin responses to phenylalanine and tyrosin in phenylketonuria. Metabolism. 1992;41:518-21.

15. Krause W, Halminski M, McDonald L, Dembure P, Salvo R, Freides D, Elsas L. Biochemical and neuropsychological effects of elevated plasma phenylalanine in patients with treated phenylketonuria. A model for the study of phenylalanine and brain function in man. J Clin Invest. 1985;75:40-8.

16. Schulpis $\mathrm{KH}$, Papakonstantinou E, Michelakakis $\mathrm{H}$, Theodoridis Th, Papandreou UR, Constantopoulos A. Elevated serum prolactin concentration in phenylketonuric patients on a 'loose diet'. Clin Endocrinol. 1998;48:99-101.

17. van Spronsen FJ, van Dijk T, Smit GP, van Rijn M, Reijngoud DJ, Berger R, Heymans HS. Large daily fluctuations in plasma tyrosine in treated patients with phenylketonuria. Am J Clin Nutr. 1996;64:916-21.
18. Landvogt C, Mengel E, Bartenstein P, Buchholz HG, Schreckenberger $M$, Siessmeier T, Scheurich A, Feldmann R, Weglage J, Cumming P Zepp F, Ullrich K. Reduced cerebral fluoro-L-dopamine uptake in adult patients suffering from phenylketonuria. J Cereb Blood Flow Metab. 2008;28(4):824-31.

19. Wang KD, Zhou ZS, Shen M, Hong W, Yu WM. Quantification of phenylalanine in the brain of patients with hyperphenylalaninemia by (1) $\mathrm{H}$ magnetic resonance spectroscopy. Zhonghou Er Ke Za Zhi. 2009:47(2):119-23.

20. Weglage J, Wiedermann D, Denecke J, Feldmann R, Koch HG, Ullrich K, Möller HE. Individual blood-brain barrier phenylalanine transport in siblings with classical phenylketonuria. J Inherit Metab Dis. 2002;25(6):431-6.

\section{Submit your next manuscript to BioMed Central and we will help you at every step:}

- We accept pre-submission inquiries

- Our selector tool helps you to find the most relevant journal

- We provide round the clock customer support

- Convenient online submission

- Thorough peer review

- Inclusion in PubMed and all major indexing services

- Maximum visibility for your research

Submit your manuscript at www.biomedcentral com/submit 